\title{
Industrial survey of ISO surface texture parameters
}

\author{
L D Todhunter ${ }^{\mathrm{a}, *}$, R K Leach ${ }^{\mathrm{a}}$, S D A Lawes ${ }^{\mathrm{a}}$, F Blateyron ${ }^{\mathrm{b}}$ \\ ${ }^{a}$ Manufacturing Metrology Team, Faculty of Engineering, University of Nottingham, UK \\ ${ }^{b}$ Digital Surf, Besançon, France
}

\begin{abstract}
Results of an international survey are presented, detailing the use of surface texture parameters in industry. The survey received 179 responses from a total of 34 countries, revealing the use of a variety of parameters from ISO 4287, ISO 12085, ISO 13565-2/3 and ISO 25178. The survey responses show an increase in the number of users of profile parameters, and an increase in the range of surface texture parameters used, compared to the results from a similar survey in 1999, as well as a significant uptake of the new areal surface texture parameters. Individual sector usage is also discussed.

Keywords: surface texture, profile parameters, areal parameters
\end{abstract}

\section{Introduction}

The analysis of surface texture is an increasingly important aspect of manufacturing industry. As manufactured parts get more complex, tolerances get tighter and the need for accurate control of surface texture becomes more prominent [1]. Surface texture parameters facilitate surface control by assigning the surface a quantitative value, calculated via a series of mathematical operations $[2,3]$, that enable comparisons to be made with other surfaces, surfaces to be specified on engineering drawings and functional information to be quantified $[4,5]$.

In 1999, a survey was conducted by CIRP to identify the surface texture parameters used in industry [6]. The survey obtained a total of 284 responses, shedding light on the use of profile parameters from current ISO

\footnotetext{
*Corresponding author

Email address: luke.todhunter@nottingham.ac.uk (L D Todhunter)
} 
specification standards of the time: ISO 4287 [2], ISO 12085 [7], and ISO 13565-2 [8].

In the seventeen years since the publication of the 1999 survey, much has changed, with the most important advancement being the introduction of areal surface texture parameters [9, 10], as described in ISO 25178-2 [11]. In addition, commercial software packages have been released for the calculation of surface texture parameters and it is, therefore, expected that industry is starting to embrace areal surface texture characterisation. Optical instruments have also become increasingly popular in industry, which are often areal in nature $[4,12]$.

These factors bring to light the need for a new surface texture parameter survey, to identify the new usage patterns of surface texture parameters that are born from the new instruments and software that are becoming more popular in industry. Additionally, a new surface texture parameter survey will give insight into the uptake of the areal surface texture parameters of ISO 25178-2, since its publication in 2012.

\subsection{Surface texture parameter survey}

This study used an on-line based survey to obtain information from voluntary respondents about the surface texture parameters that they use. Invitations to complete the survey were sent out by email to suitable correspondents. The survey was mainly aimed at CIRP industry contacts, as this provided opportunity for responses from a wide range of relevant disciplines. The survey was later expanded to a much wider audience to increase the number of responses, for example by advertising the survey to conference attendees. The survey included parameters from ISO 4287, ISO 12085 and ISO 13562-2, similar to the 1999 survey, but also included ISO 13565-3 [13] and areal parameters from ISO 25178-2. In addition, the survey requested details about the respondents' company, and also gave the option for respondents to share their opinions on the current selection of surface texture parameters available. A copy of the online form in included as an appendix.

The survey was open to responses for eight months, from March 2016 to November 2016, and obtained a total of 179 responses from a variety of industrial users spread internationally across thirty-four countries. The distribution of responses is shown in Table 1. It should be noted here that 179 responses is a relatively small number, and consequently the conclusions that can be drawn from the survey results are limited. That said, the responses come from a variety of disciplines and countries, and serve as a 


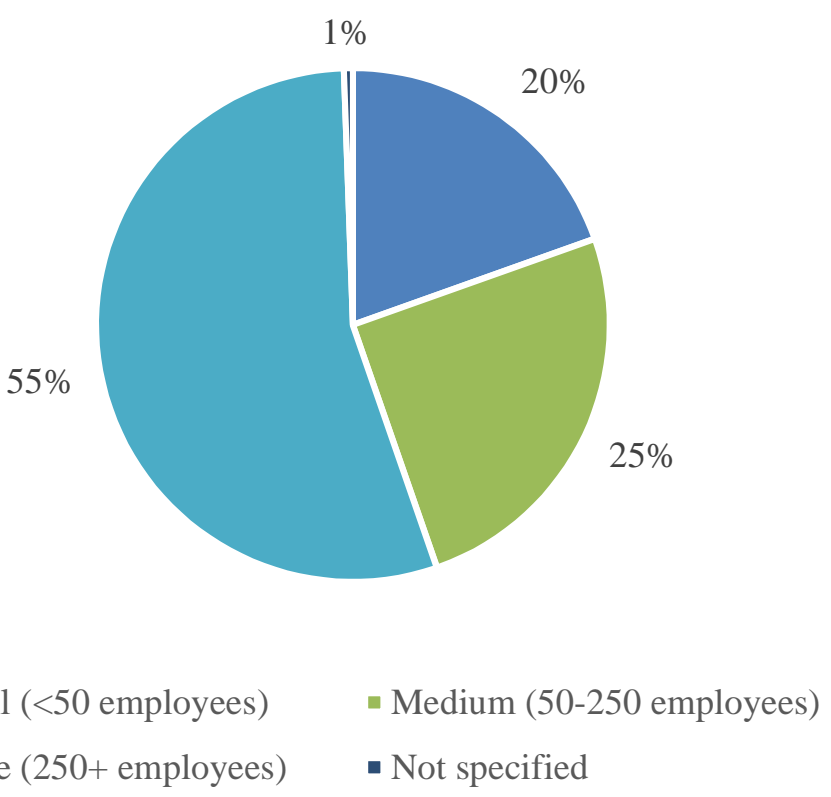

Figure 1: The percentage of responses to the survey with a given company size.

viable sample from which to learn about current surface texture parameter use, delivering a useful update to the original 1999 survey.

\section{Results}

\subsection{Participant details}

Figure 1 shows the company sizes of the participants, in terms of number of employees. The majority of responses were from large companies, with $55 \%$. This is in contrast to the 1999 survey, in which medium sized companies gave the most responses with $45 \%$.

Information about the participants' instrument usage is shown in Figure 2. The results indicate that whilst contact instruments are still popular, optical instruments have seen significant adoption, with $66 \%$ of respondents using them either exclusively or in conjunction with contact instruments. As optical instruments are often areal in nature, it comes as no surprise that areal instrument modes have seen similar adoption, again with $66 \%$ using some form of areal instrument mode.

Figure 3 shows how the survey responses were split in terms of industry sector. The most responses for this survey came from research institutions, such as universities. These operate in many of the other disciplines listed, 
Table 1: List of participating countries and the number of responses from each.

\begin{tabular}{|c|c|}
\hline Country & Responses \\
Algeria & 1 \\
Austria & 1 \\
Belgium & 3 \\
Brazil & 3 \\
Canada & 1 \\
China & 4 \\
Czech Republic & 1 \\
Denmark & 11 \\
Finland & 1 \\
France & 11 \\
Germany & 22 \\
Honk Kong & 1 \\
Hungary & 1 \\
India & 5 \\
Ireland & 1 \\
Israel & 1 \\
Italy & 7 \\
Japan & 1 \\
Japan & 1 \\
South Korea & 5 \\
Latvia & 1 \\
Luxembourg & 1 \\
Mexico & 1 \\
Netherlands & 4 \\
Poland & 1 \\
Portugal & 1 \\
Russia & 1 \\
South Africa & 1 \\
Spain & 7 \\
Sweden & 12 \\
Switzerland & 9 \\
United Kingdom & 17 \\
United States & 40 \\
Not specified & 1 \\
Total & 179 \\
\hline
\end{tabular}



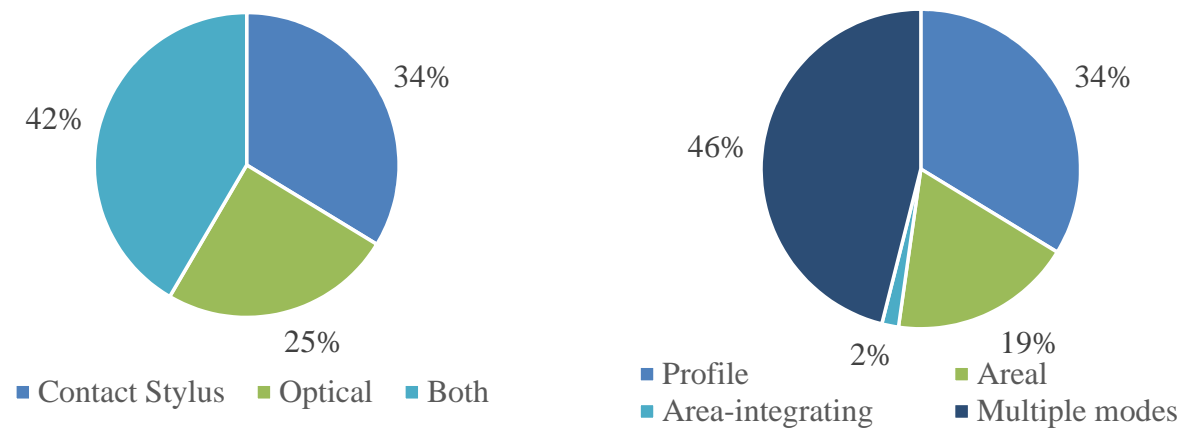

Figure 2: Left: Instrument type usage as a percentage of responses. Right: Instrument mode usage as a percentage of responses.

but focus on none in particular. Other sectors that gave a notable number of responses were 'metrology \& calibration', 'automotive \& aerospace' and 'product manufacturing'. The 'product manufacturing' category is a broad sector encompassing participant companies who produce consumer or specialist goods or materials, but do not specifically operate in one of the other industrial sectors. Several of the sectors here are comprised of only a few participants, such as 'tribology' and 'archaeology \& anthropology', and as such are poorly represented. Individual analysis of these sectors would not provide useful information, and consequently they have been omitted from any individual analyses.

\subsection{Profile surface texture parameters}

Before discussing the adoption of the recently introduced areal surface texture parameters, it is first useful to analyse the profile case. As many of the profile parameters were the subject of the 1999 survey, this analysis has the ability to compare the current profile parameter usage to that of 1999, and obtain insight into the evolution of parameter usage in industry.

\subsubsection{ISO 4287 profile parameters}

Figure 4 gives the survey responses for the usage of the ISO 4287 profile surface texture parameters for both 2016 and 1999, as a percentage of the total number of responses. For example, $15 \%$ of the 179 participants in the 2016 survey used the $P z$ parameter, compared to $<5 \%$ of the 284 participants in the 1999 survey. The most immediate conclusion to draw from the results is the unanimous increase in parameter usage across all parameters. 


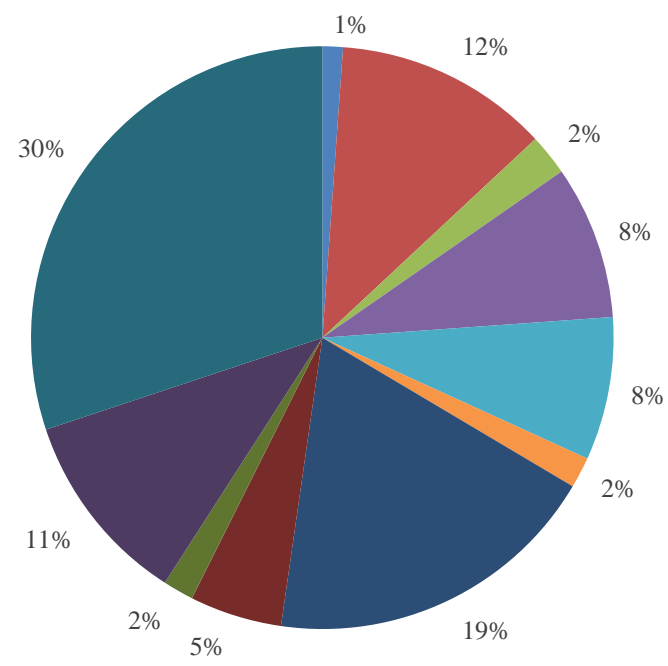

- Archaeology \& Anthropology

- Automotive \& Aerospace

- Defence \& Energy

- Electronics

- Machining \& Tooling

- Tribology

- Metrology \& Calibration

-Optics

- Pharma \& Chemical

- Product Manufacturing

- Research Institutions

Figure 3: Industry sectors represented in the survey as a percentage of total number of responses.

Such a result shows the increased awareness of surface texture parameters in industry, and their more widespread use.

As expected, the $R a$ parameter and the primary/waviness profile equivalents remain the most popular parameter used, however, since 1999 a significant relative increase is seen for the less well-known parameters, such as the skewness and kurtosis parameters $X s k$ and $X k u$. This indicates a much greater understanding of the surface texture parameters as a whole, and their individual uses. This being said, it should be considered that this widespread adoption of all surface texture parameters could be due to the exponential increase in computational power available to measurement instrument users since 1999, allowing them to calculate all parameters with relative ease. This blanket approach to parameter selection would unfortunately, therefore, indicate no further understanding of individual surface texture parameters than the results obtained in 1999. This over-abundance of calculated parameter values has been termed 'parameter rash' [4], and serves to deliver no more insight into the required information from a surface measurement than the use of no parameters. Instead, parameter values should be displayed with an intended purpose.

An interesting observation from the results is the significant increase in the use of waviness parameters compared to 1999. With the exception of 

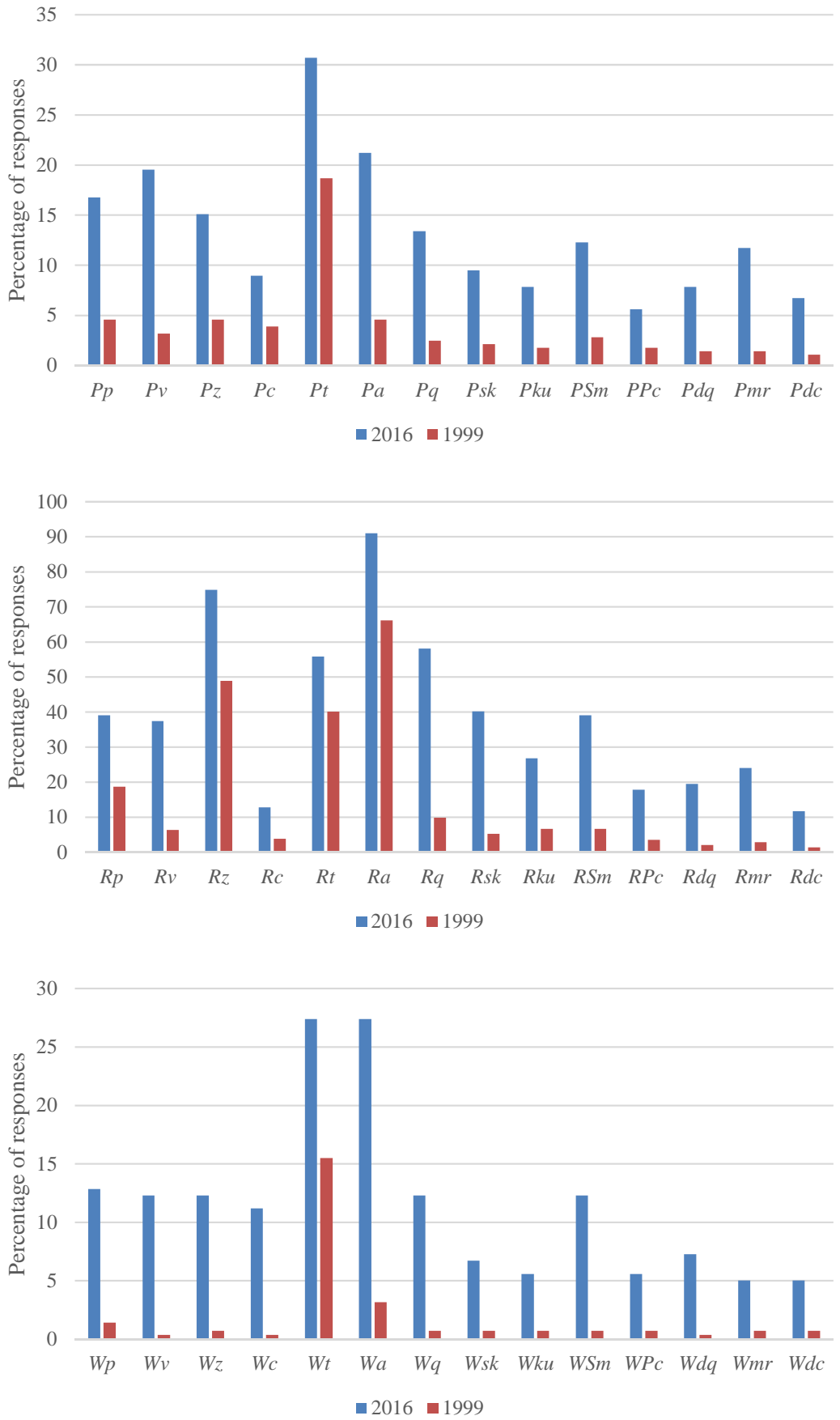

Figure 4: Responses that indicated use of ISO 4287 parameters for the 2016 (blue) and 1999 (red) surveys, given as the percentage of the total number of responses that use each parameter. Top: Primary parameters, Middle: Roughness parameters. Bottom: Waviness parameters. 
the $W t$ parameter, which was used by $~ 15 \%$ of participants, most waviness parameters were hardly used in 1999, with only $1 \%$ of participants indicating parameter use. The new results show a much greater uptake, with waviness parameters on average used by $12 \%$ of participants.

Figure 5 shows the 2016 results for the ISO 4287 profile parameters split into individual sectors. Whilst the same overall trends are visible for each sector as for all participants, some results of interest for individual sectors can be seen. The most obvious result is the high uptake of almost all parameters by the 'metrology and calibration' sector. This is to be expected; the 'metrology and calibration' sector has a specific focus on characterising a surface measurement as completely as possible, and so it comes as no surprise that the sector would use a wide variety of parameters. This trend continues throughout the results of this paper.

Ignoring the roughness parameters, another interesting result is that the 'automotive and aerospace' sector shows quite high usage for the $P t$, $W t$ and $W a$ parameters, but very low usage for the rest. This small variety of parameters used suggests the sector has identified a small selection of parameters that are useful, and avoided the so-called 'parameter rash' [4] by only using those that are necessary.

\subsubsection{ISO 12085 motif parameters and ISO 13565-2/3 stratified surface parameters}

Figure 6 and Figure 8 give the survey results of the usage of profile surface texture parameters given in ISO 12085 and ISO 13565-2/3 respectively.

As seen for the ISO 4287 parameters, ISO 12085 has seen an increase in use for all parameters. In particular, the $R, W$ and $P t$ parameters have seen a substantial increase in use, although the number of users is still low compared to the ISO 4287 parameters. Parameters $R$ and $W$ indicate the mean depths of the roughness and waviness motifs, respectively, and $P t$ is the peak-to-valley height of the roughness motifs. These are all relatively simple to calculate, yet valuable parameter definitions, so it is understandable that these are the most adopted parameters from the standard.

It should be noted here that the 1999 survey only included the first seven parameters shown in figure 6 , as these are the only ones to be included in the ISO 12085 standard document. The 12085 parameters were originally defined in a French automotive standard, but not all parameters were transferred to the ISO document [14]. The rest are still featured in many third-party surface texture parameter calculation software packages, and so were included in this survey. 

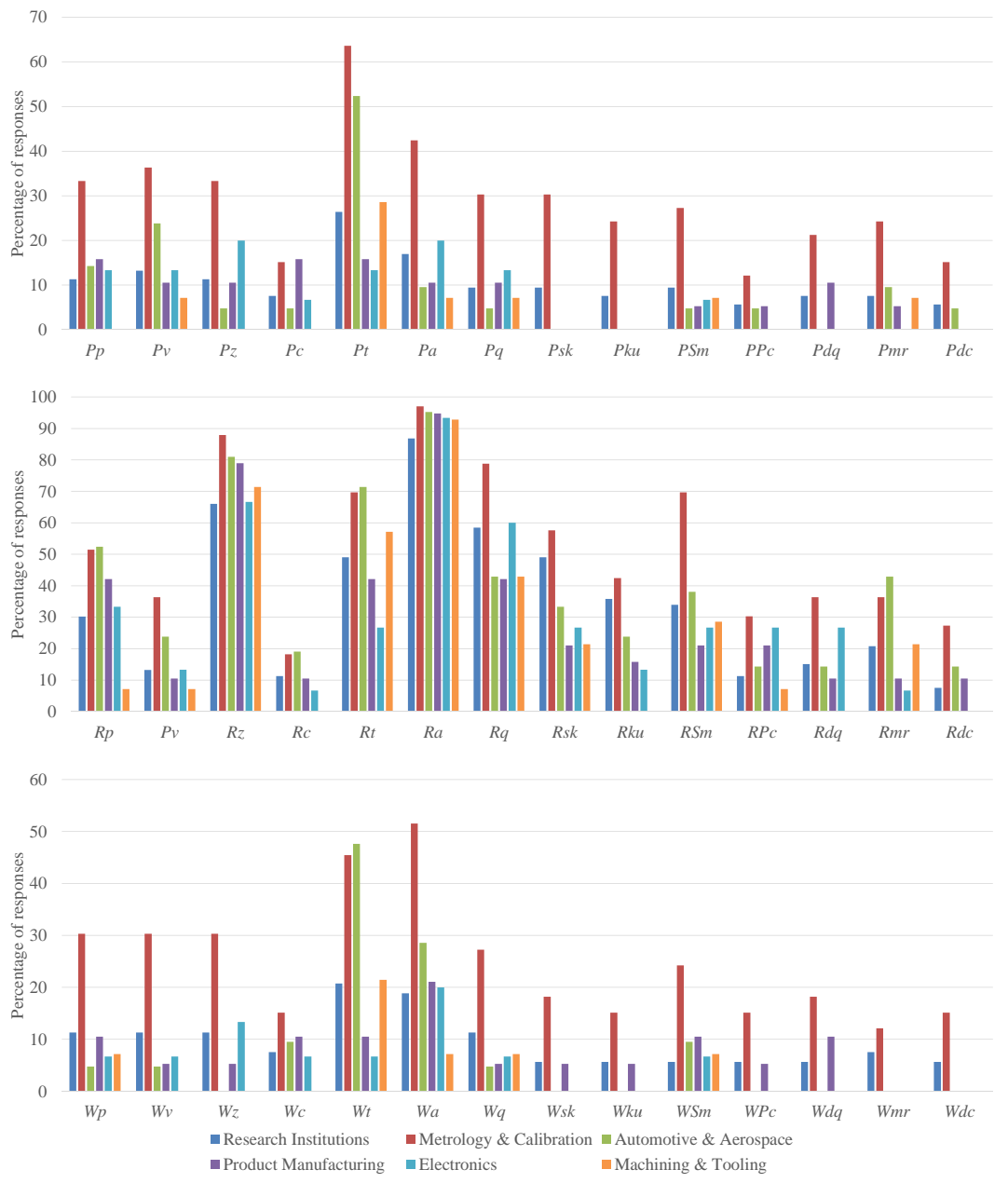

Figure 5: Percentage of responses that indicated the use of ISO 4287 parameters, displayed for individual sectors. Top: Primary parameters. Middle: Roughness parameters. Bottom: Waviness parameters. 


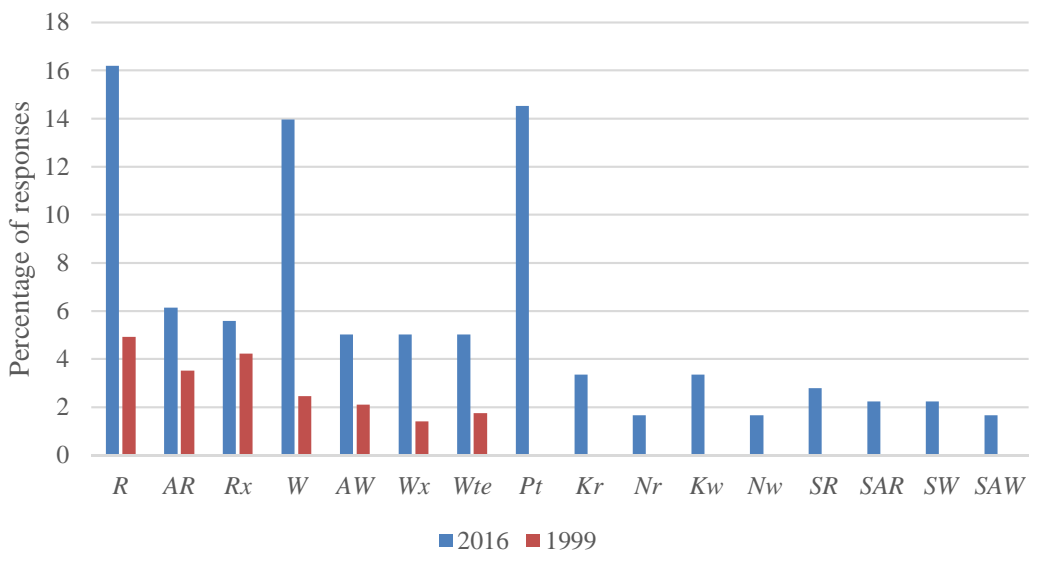

Figure 6: Percentage of responses that indicated use of ISO 12085 motif parameters for the 2016 (blue) and 1999 (red) surveys.

The individual sector results for the ISO 12085 motif parameters are given in figure 7 . Here, several of the motif parameters are shown to be used by over $22 \%$ of the 'electronics' sector, suggesting the 'electronics' sector finds characterisation of surface motifs more useful than other sectors.

The survey results for the ISO 13565 parameters show a significant increase in the use of all parameters. An interesting point is that in 1999 , there is a fairly even uptake of the parameters, with each one used by ${ }^{\sim} 10 \%$ of participants. This has changed somewhat in 2016, where the MR1 and $M R 2$ parameters are used less, and the $R p k$ parameter has become the most popular. The four ISO 13565-3 parameters were not included in the 1999

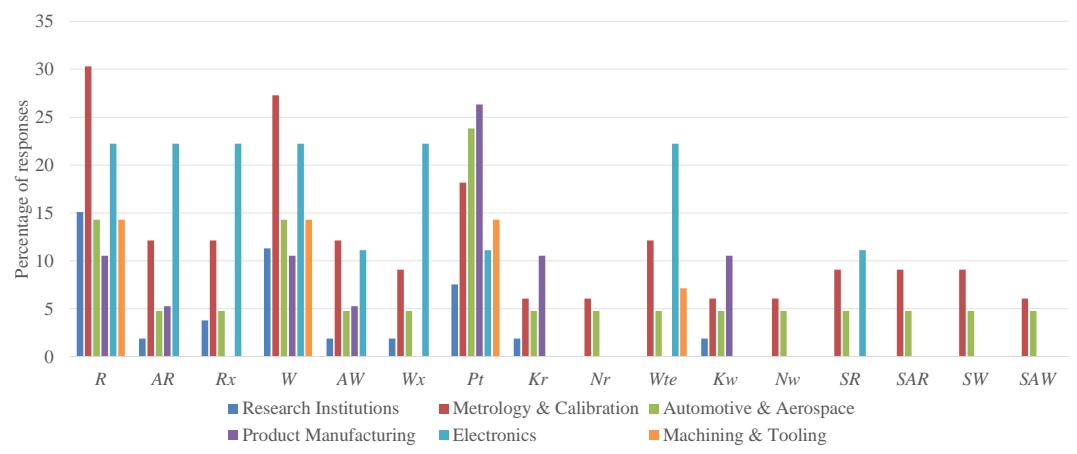

Figure 7: Percentage of responses that indicated use of ISO 12085 motif parameters, split into individual sectors 


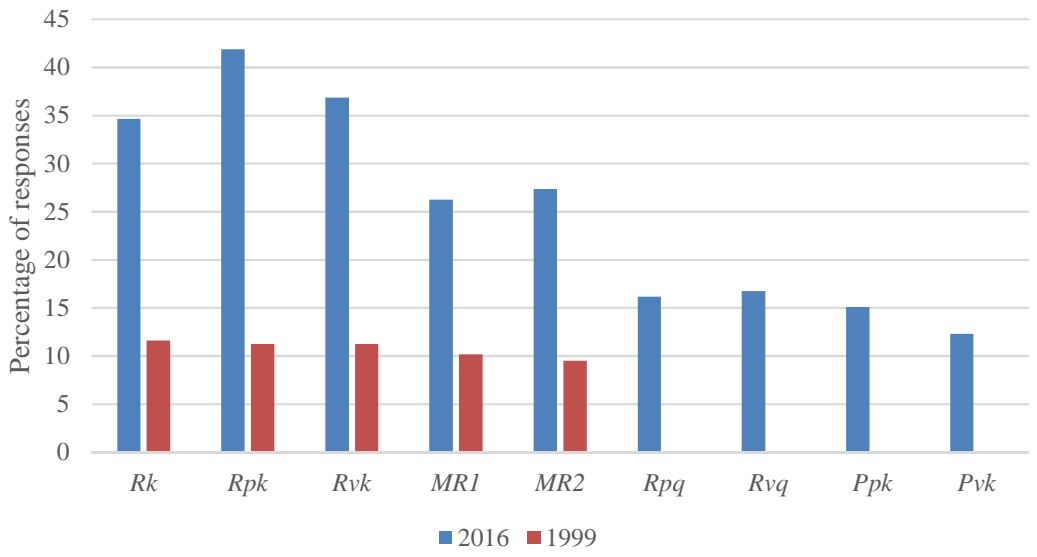

Figure 8: Percentage of responses that indicated use of ISO 13565-2/3 stratified surface parameters for the 2016 (blue) and 1999 (red) surveys.

survey and so are not able to be compared; however, it is clear that these are less popular than the slightly older parameters found in part 2 .

Figure 9 shows the individual sector results for the stratified surface parameters of ISO $13565-2 / 3$. As mentioned above, the ISO 13565-2 parameters are the more popular of the two sets, especially with the 'electronics', 'automotive and aerospace' and 'metrology and calibration' sectors. The 'machining and tooling' also displays a notable uptake of these parameters, albeit not as strongly as those aforementioned. These standards focus on characterising stratified surfaces, created through two processes, such as machining and polishing, and so these parameters are of use for sectors that utilise such techniques.

\subsection{ISO 25178-2 Areal surface texture parameters}

Figure 10 gives the survey results for the field, feature and functional areal surface texture parameters given in ISO 25178-2. These parameters were published in 2012, much more recently than many of the other parameters featured in this survey, and as a result, no comparisons can be made with the 1999 survey. Areal surface texture parameters operate in an additional dimension to the profile parameters that precede it. This is a large step forward in the field of surface texture analysis, and thus the results of this survey deliver an interesting insight into the industry's adoption of a new era of surface analysis. 


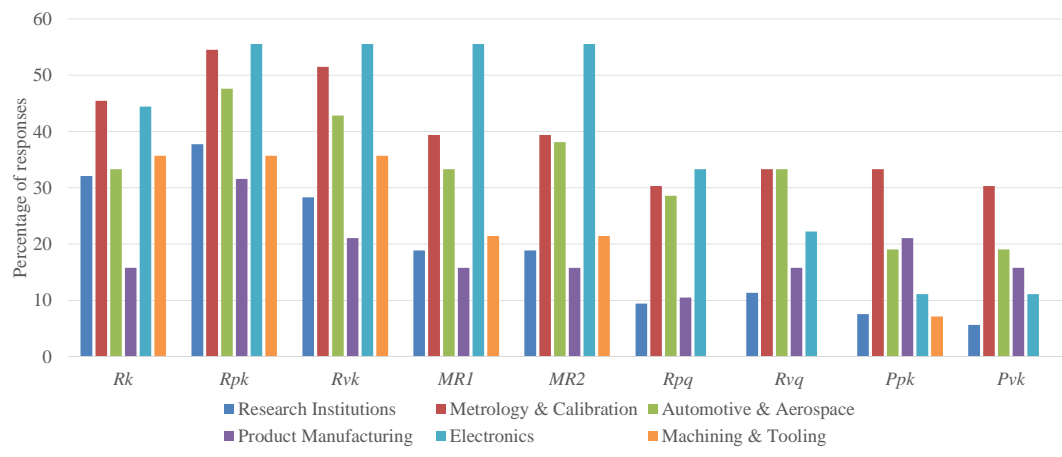

Figure 9: Percentage of responses that indicated use of ISO 13565-2/3 stratified surface parameters, split into individual sectors

The ISO 25178-2 parameters are split into three categories: field, feature and functional parameters. The field parameters share the greatest similarity with the ISO 4287 profile parameters, and so it comes as no surprise that these have seen the largest uptake by industry, with an average of $31 \%$ of participants claiming use. Unsurprisingly, the areal equivalents of $R a, R z$ and $R q$, some of the most popular profile parameters, are the most popular areal parameters, with $S a$ being used by $56 \%$ of participants. Interestingly, $S t$ shows one of the lower adoptions at $20 \%$, despite its profile equivalent $R / P / W t$ being one of the most used profile parameters.

The ISO 25178-2 feature parameters focus on areal feature identification, a new type of surface analysis with no real parallel in the profile world. As a result, these are the least used of the three categories of areal surface parameters. That being said, they are still used by a notable amount, ranging from $~ 6 \%$ to $13 \%$, which is more than the majority of motif parameters found in ISO 12085. Similar to the discussion in section 2.2.1, part of the reason for these usage results could be due to the tendency to simply calculate all available parameters and identify useful parameter correlations afterwards, as computational power and third-party parameter calculation software are now readily available.

The functional parameters revolve around the concept of the material ratio of the surface, and variations thereof. This was introduced in ISO 4287, and so is a surface analysis method familiar to industry. As a result, these parameters have been used by an appreciable percentage of participants, with most ranging from $10 \%$ to $20 \%$ adoption.

Overall, the ISO 25178-2 areal parameters have been used by a large 

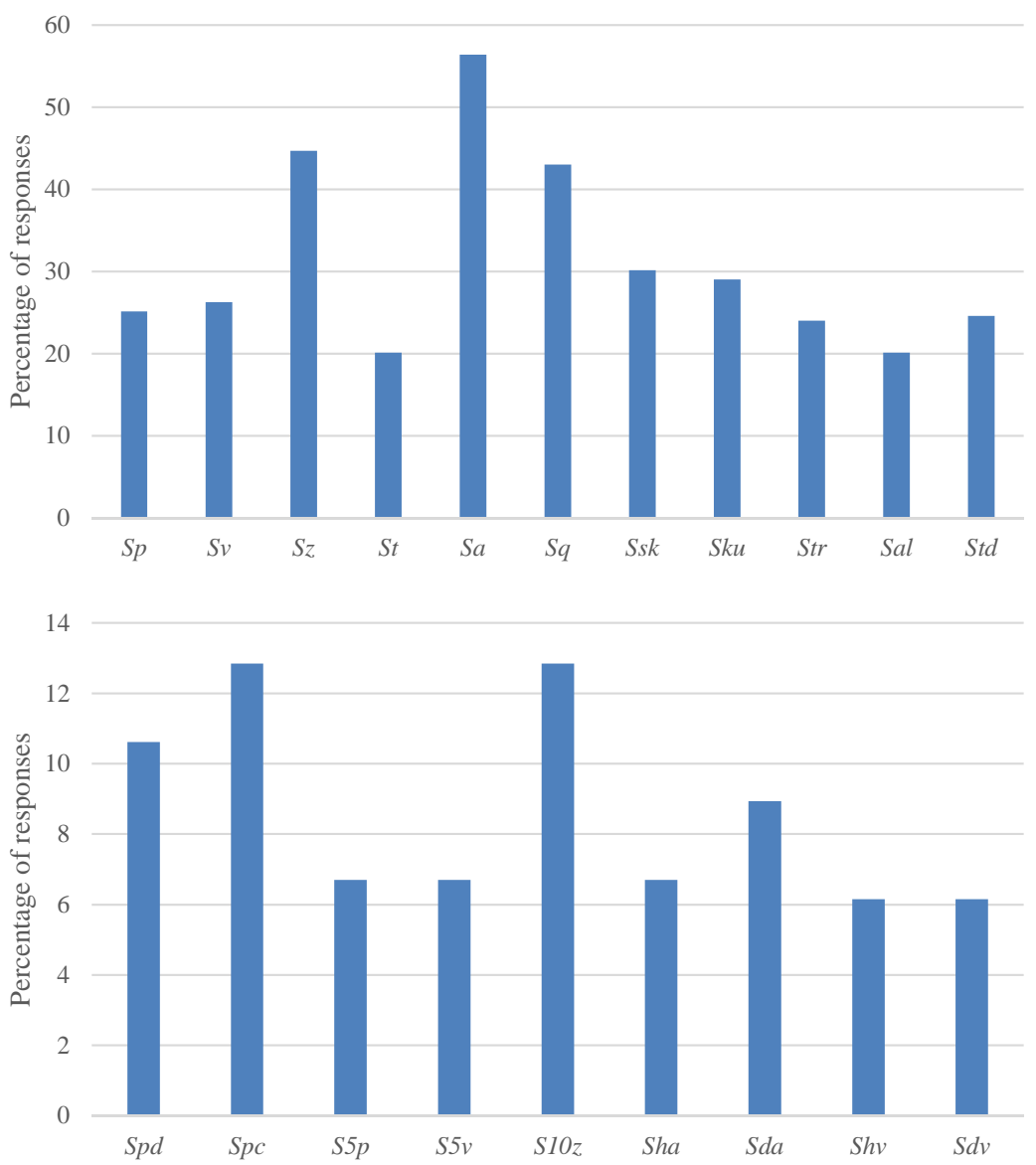

25

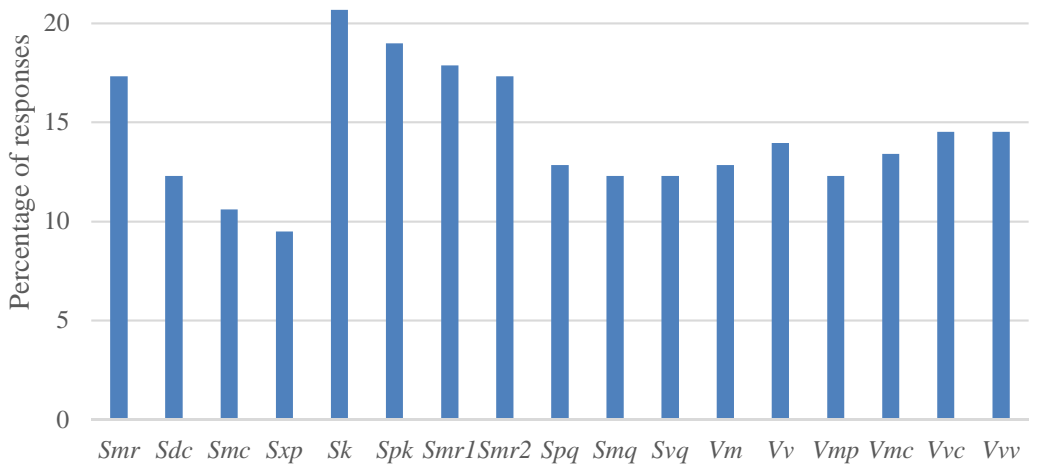

Figure 10: Percentage of responses that indicated use of ISO 25178-2 parameters for the field (top), feature (middle) and functional (bottom) parameters. Note that there are no 1999 results to display here as the parameters were not defined at that time. 
number of participants considering their age and their differences from the old profile parameters. The inclusion of several analogous parameters to those used for profile surfaces has probably enabled a smoother transition into the areal era, and has allowed almost $60 \%$ of participants to perform some form of areal surface texture analysis.

Figure 11 gives the individual sector results for the ISO 25178-2 parameters. Aside from the previously mentioned widespread use by the 'metrology and calibration' sector, the results also show significant adoption of the areal parameters by the 'research institution' sector. This sector is comprised primarily of universities and other laboratories that conduct scientific research, so it is understandable that this sector would be among the first to adopt the latest parameters and methods available to conduct their research.

The areal field parameters also see adoption by the 'electronics' and 'product manufacturing' sectors. The 'automotive and aerospace' and, to a lesser extent, 'machining and tooling' sectors, however, show little use of these parameters. These is somewhat expected, as these are the two older industrial sectors of the group, and rely on more traditional manufacturing and engineering methods. These results suggest it would be useful for these sectors to receive further education on the latest areal surface parameters to fully utilise the new surface information that can be obtained.

For the functional and feature parameters, the individual sector adoption is less impressive. The 'electronics' and 'machining and tooling' sectors show come adoption of a small range of parameters, in particular those which have a profile analogue, such as Smr. For the majority of the parameters, however, adoption is poor. It is clear that aside from the 'research institution' and 'metrology and calibration' sectors, the majority of participants do not see a use for the functional and feature parameters. Further education and guidance is required to give these sectors an understanding of what these new parameters can offer.

\subsection{Additional information}

In addition to selecting which parameters they used from a predefined list, participants were also encouraged to give their thoughts on the current ISO parameter selection. A total of sixty-nine comments were received detailing the thoughts of participants on the current state of surface texture parameters. Though these responses were varied, several key points came up.

A common comment given by participants was a lack of in-depth understanding of what each parameter means, and what it tells the user about 

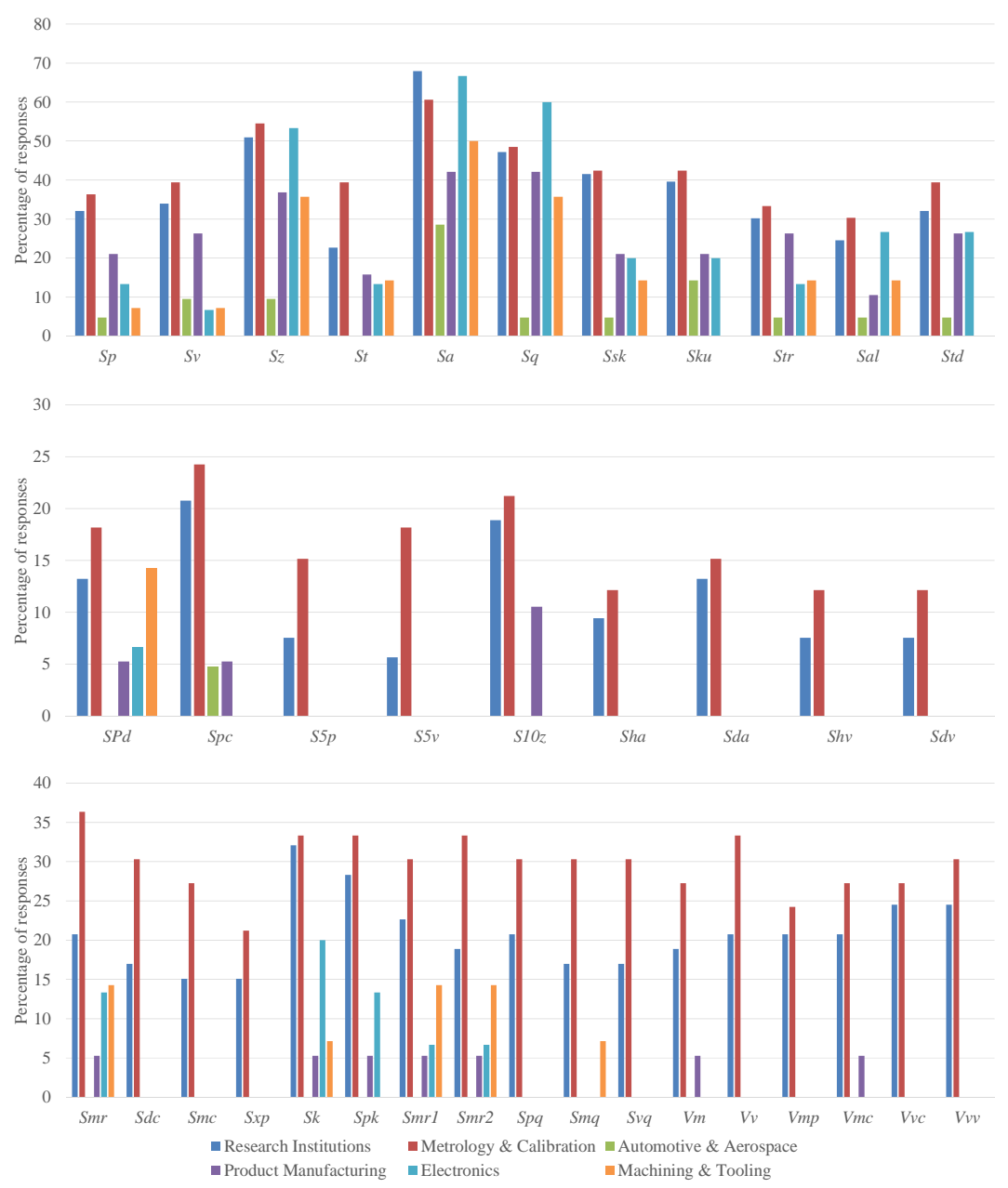

Figure 11: Percentage of responses that indicated use of ISO 25178-2 parameters for the field (top), feature (middle) and functional (bottom) parameters, split into individuals sectors. 
the surface that is different from any other parameter. Several comments called for further education and training on the current parameter selection, or a more in-depth explanation of the parameter in the ISO specification standard definition, with the hope to aid this understanding and tailor the parameter values calculated to the measurement purpose. These comments suggest that new parameters are published with little further explanation to users regarding their specific uses and differences, which is something that would be of use to parameter users in industry.

Another comment given comes from participants that only use profile surface texture parameters, mentioning that they know of no manufacturers that are using areal parameters. This lack of awareness of the usage of areal parameters suggests there are some fields in industry that are yet to adopt areal parameters, and are still limited to older profile parameters. Further education is required on areal surface texture parameters in these fields to promote areal parameter use.

A final popular comment from participants is the need for more areal parameters, particularly for characterising specific features on the surface. Several of these comments make mention of additive manufacturing, and how the resulting surfaces from some of those processes contain features that do not conform well to the current selection of parameters (see Townsend for a recent review on this subject [15]). As areal surface measurements and new manufacturing techniques gain popularity, better education and guidance in good practice are required for surface texture parameters, allowing users to link surface texture parameters with process parameters and function, and better understand the parameter results and the information they give about the measured surface. Of course, in contrast to these comments, several comments were received explaining that there are already too many parameters, and that these should be reduced down to a small number of key parameters, to avoid the so-called 'parameter rash' [4].

\section{Conclusions}

This survey was produced with the intention of obtaining an up-todate understanding of the usage of surface texture parameters in industry. The survey was intended to be a follow-on from the work carried out by De Chiffre in 1999, to identify the evolution of profile surface texture parameter use, and to see how the new areal surface texture parameters have been adopted. 
The survey obtained a total of 179 responses from industrial companies spread internationally across thirty-four countries and a variety of sectors. The results showed a relative increase in the use of virtually all parameters in comparison to 1999, showing a marked improvement in the uptake and importance of surface texture parameters in industry. It should be considered that this could be a consequence of the exponential increase in computational power available to users since 1999, along with greater availability of third party surface texture parameter calculation software, enabling the calculation of many more parameters with relative ease; this scenario does not necessarily mean a greater understanding of the parameters used.

In addition, this survey gave insight into the adoption of areal surface texture parameters in industry, and resulted in a significant proportion, $\sim 30 \%$ for areal field parameters, of participants indicating regular use of areal surface texture parameters. This relatively fast adoption rate indicates the value that areal parameters have in industry, and the additional layer of information that they can deliver to users that the older profile parameters cannot. A deeper look into these adoption rates, however, showed that it was mainly the 'research institutions' and 'metrology and calibration' sectors that have shown adoption of the areal parameters, and that these new parameters are yet to be used by the other sectors in industry. Further education is required for the areal parameters to allow all sectors to engage with them.

Through the inclusion of the opportunity for participants to share their thoughts on the current state of surface texture parameters available, additional conclusions can be drawn. The comments highlighted a need for further education on the surface texture parameters available to allow for more meaningful analysis, as well as the need to be able to better characterise surface features that are more relevant to new manufacturing processes, such as additive manufacturing. Additionally, these comments detail gaps in the industry where some fields have not been suitably educated on areal surface texture parameters, leading to no adoption. Further education and good practice guidance is required here to widen the appeal of areal surface texture parameters and promote parameter use.

In summary, this survey has delivered an updated view on the current usage of surface texture parameters in industry, and has shone light on potential areas for improvement in the ISO specification standards and the accompanying education. 


\section{Acknowledgements}

We would like to thank the Engineering and Physical Sciences Research Council (EPSRC Grants EP/M008983/1 and EP/1017933/1). For access to the data from this survey, please contact the author at luke.todhunter@nottingham.ac.uk.

\section{References}

[1] A. A. G. Bruzzone, H. L. Costa, P. M. Lonardo, D. A. Lucca, Advances in engineered surfaces for functional performance, Ann. CIRP 57 (2008) 750-769.

[2] ISO 4287, Geometrical Product Specifications (GPS) - Surface texture: Profile method - Terms, definitions and surface texture parameters, Geneva: International Organisation for Standardization, 1998.

[3] ISO 4288, Geometric Product Specifications (GPS) - Surface texture: Profile method - Rules and procedures for the assessment of surface texture, Geneva: International Organisation for Standardization, 1998.

[4] D. J. Whitehouse, Handbook of Surface Metrology, Institute of Physics, Bristol, 1994.

[5] R. K. Leach, Fundamental Principles of Engineering Nanometrology, 2nd Edition, Elsevier, Amsterdam, 2014.

[6] L. De Chiffre, Industrial survey on ISO surface texture parameters, Ann. CIRP 48 (3) (1999) 74-77.

[7] ISO 12085, Geometric Product Specifications (GPS) - Surface texture: Profile method - Motif Parameters, Geneva: International Organisation for Standardization, 1997.

[8] ISO 13565-2, Geometric Product Specifications (GPS) - Surface texture: Profile method - Surfaces having stratified functional properties, Geneva: International Organisation for Standardization, 1997.

[9] R. K. Leach, Characterisation of Areal Surface Texture, Springer, Berlin, 2013.

[10] L. Blunt, X. Jiang, Advanced Techniques for Assessment Surface Topography: Development of a Basis for 3D Surface Texture Standards "SURFSTAND", Kogan Page Science, London, 2003.

[11] ISO 25178-2, Geometrical Product Specifications (GPS) - Surface texture: Area Part 2: Terms, definitions and surface texture parameters, Geneva: International Organisation for Standardization, 2012.

[12] R. K. Leach, Optical Measurement of Surface Topography, Springer, Berlin, 2011.

[13] ISO 13565-3, Geometrical Product Specifications (GPS) Surface texture: Profile method - Surfaces having stratified functional properties Part 3: Height characterization using the material probability curve, Geneva: International Organisation for Standardization, 2000.

[14] CNOMO E00.14.015.N, Etats geometriques de surface calcul des parametres de profil, 1993.

[15] A. Townsend, N. Senin, L. Blunt, R. K. Leach, J. S. Taylor, Surface texture metrology for metal additive manufacturing : a review, Precision Engineering 46 (2016) $34-47$. 


\title{
Appendix A. Copy of online parameter survey form
}

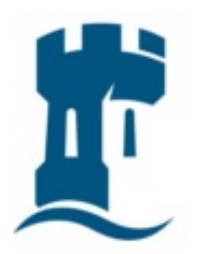

The University of Nottingham

\author{
UNITED KINGDOM $\cdot$ CHINA $\cdot$ MALAYSIA
}

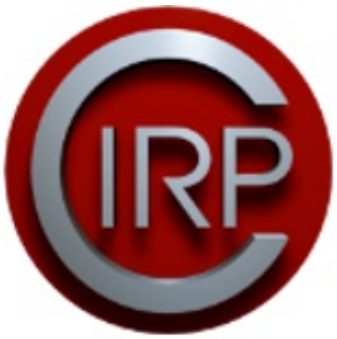

\section{CIRP Parameter Survey}

\section{Page 1: Information}

In 1999, CIRP conducted an industrial survey of the use of surface texture parameters. In the 17 years since, much has changed, with the most important advancement being the introduction of areal surface texture parameters as described in ISO 25178-2. There has also been the release of commercial software packages for the calculation of surface texture parameters and, therefore, it is expected that industry is starting to embrace areal surface texture characterisation. Industry is also increasingly using more optical instruments, which are often inherently areal in nature. These factors bring to light the need for a new parameter survey, to investigate whether industry really has been adopting areal surface texture parameters. The results of the survey will be published and presented at a forthcoming CIRP meeting.

Professor Richard Leach, Dr Simon Lawes and Mr Luke Todhunter Manufacturing Metrology Team

Advanced Manufacturing Research Group

Faculty of Engineering

University of Nottingham

Questions about the survey should be addressed to richard.leach@nottingham.ac.uk.

The survey will take no more than 10 minutes to complete.

All responses will be treated as confidential. 
Please take the time to fill in the survey below

If you wish to view the full survey before completing it, feel free to download a .PDF copy of the survey by clicking here.

1. Company * Required

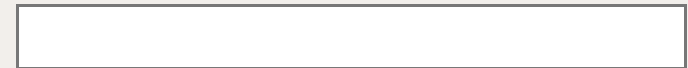

2. Address * Required

\section{Phone * Required}

4. Email * Required

$2 / 8$ 


\section{Contact Person}

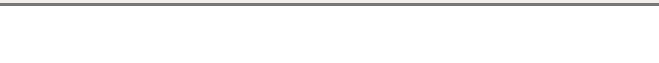

6. Company size

$\begin{array}{ccr}\text { Small }(<50 & \sim \text { Medium }(50-250 & \sim \\ \text { employees }) & \text { employees }) & \text { employees })\end{array}$

7. Department

c Development $\quad$ Production Quality

c Other

8. Type of instrument used

$\sim$ Contact Stylus $\sim$ Optical Both

O Other

8.a. If you selected Other, please specify:

9. Instrument mode used 
c Profile

c Areal

c Multiple modes
C Area-integrating (e.g. scattering) 


\section{Page 2: Parameters used}

In the sections below, please mark the parameters used in your company.

10. Parameters defined in ISO 4287: 1997
$\ulcorner\mathrm{Pp}$
$\ulcorner\mathrm{Rp}$
$\ulcorner W p$
$\Gamma \mathrm{Pv}$
$\ulcorner\mathrm{Rv}$
$\ulcorner W v$
$\Gamma \mathrm{Pz}$
$\ulcorner\mathrm{Rz}$
$\ulcorner\mathrm{Wz}$
$\ulcorner\mathrm{Pc}$
$\ulcorner\mathrm{Rc}$
$\ulcorner W c$
$\ulcorner\mathrm{Pt}$
$\ulcorner\mathrm{Rt}$
$\ulcorner\mathrm{Wt}$
$\digamma \mathrm{Pa}$
$\digamma \mathrm{Ra}$
$\ulcorner\mathrm{Wa}$
$\ulcorner\mathrm{Pq}$
$\ulcorner\mathrm{Rq}$
$\ulcorner W q$
$\ulcorner$ Psk
$\ulcorner$ Rsk
$\ulcorner$ Wsk
$\ulcorner\mathrm{Pku}$
$\ulcorner$ Rku
$\ulcorner$ Wku
$\ulcorner\mathrm{PSm}$
$\Gamma \mathrm{RSm}$
$\ulcorner\mathrm{WSm}$
$\ulcorner\mathrm{PPc}$
$\ulcorner\mathrm{RPc}$
$\ulcorner\mathrm{WPc}$
$\ulcorner$ Pdq
$\lceil\mathrm{Rdq}$
$\ulcorner$ Wdq
$\ulcorner\mathrm{Pmr}$
$\ulcorner\mathrm{Rmr}$
$\ulcorner\mathrm{Wmr}$
$\ulcorner\mathrm{Pdc}$
$\ulcorner$ Rdc
$\ulcorner$ Wdc

11. Parameters defined in ISO $12085: 1996$
$\lceil\mathrm{R}$
$\digamma \mathrm{AR}$
$\ulcorner\mathrm{Rx}$
$\ulcorner\mathrm{W}$
$\Gamma \mathrm{AW}$
$\Gamma \mathrm{Wx}$
$\ulcorner\mathrm{Pt}$
$\ulcorner\mathrm{Kr}$
$\ulcorner\mathrm{Nr}$
$\Gamma$ Wte
$\ulcorner\mathrm{Kw}$
$\ulcorner\mathrm{Nw}$
$\ulcorner\mathrm{SR}$
$\ulcorner$ SAR
$\ulcorner\mathrm{SW}$
$\Gamma$ SAW 
12. Parameters defined in ISO 16565-2: 1996 and ISO 16565-3: 1998
$\Gamma \mathrm{Rk}$
$\ulcorner$ Rpk
$\ulcorner$ Rvk
$\ulcorner\mathrm{MR} 1$
$\ulcorner$ MR2
$\ulcorner$ Rpq
$\Gamma \operatorname{Rvq}$
$\Gamma$ Ppk
$\ulcorner$ Pvk

13. Parameters defined in ISO 25178-2: 2010 - Field parameters
$\Gamma \mathrm{Sp}$
$\Gamma \mathrm{Sv}$
$\ulcorner\mathrm{Sz}$
$\Gamma \mathrm{St}$
$\Gamma \mathrm{Sa}$
$\Gamma \mathrm{Sq}$
$\Gamma$ Ssk
$\Gamma \mathrm{Sku}$
$\Gamma \mathrm{Str}$
$\Gamma \mathrm{Sal}$
$\Gamma$ Std

14. Parameters defined in ISO 25178-2: 2010 - Functional parameters
$\Gamma \mathrm{Smr}$
$\ulcorner\mathrm{Sdc}$
$\Gamma \mathrm{Smc}$
$\Gamma \operatorname{Sxp}$
$\Gamma \mathrm{Sk}$
$\Gamma \mathrm{Spk}$
$\ulcorner$ Smr1
$\Gamma \mathrm{Smr2}$
$\ulcorner$ Spq
$\ulcorner$ Smq
$\Gamma \mathrm{Svq}$
$\Gamma \mathrm{Vm}$
$\Gamma \mathrm{Vv}$
$\ulcorner\mathrm{Vmp}$
$\ulcorner\mathrm{Vmc}$
$\Gamma \mathrm{Vvc}$
$\ulcorner\mathrm{Vvv}$

15. Parameters defined in ISO 25178-2: 2010 - Feature parameters
$\Gamma \mathrm{SPd}$
$\ulcorner\mathrm{Spc}$
$\ulcorner\mathrm{S} 5 \mathrm{p}$
$\ulcorner$ S5V
$\ulcorner\mathrm{S} 10 \mathrm{z}$
$\ulcorner$ Sha
$\ulcorner$ Sda
$\ulcorner$ Shv
$\Gamma \mathrm{Sdv}$

$6 / 8$ 
16. Other parameters not defined in ISO standards (please indicate standard):

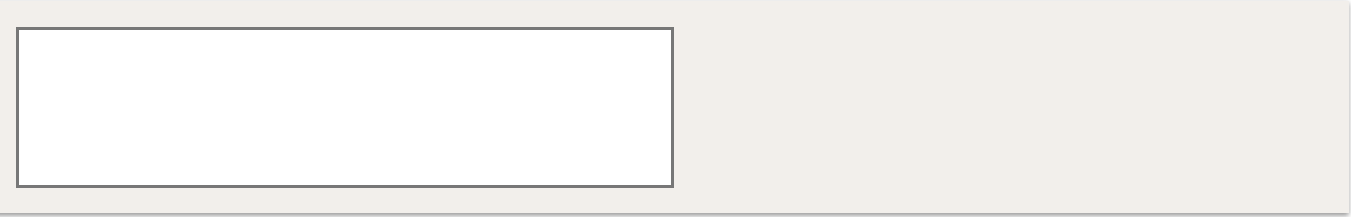

17. Please share any thoughts/opinions you have on the current range of parameters in use. 